\title{
The Reverse Hemolytic Plaque Assay in Endocrine Pathology
}

The introduction of immunocytochemistry, electron microscopy, and in situ hybridization made it possible to study endocrine tumors at cellular and subcellular levels and helped us obtain a deeper insight into the regulation of hormone secretion. Radioimmunoassay has made it possible to determine the total amount of hormone secteted under in vivo and in vitro conditions. In situ hybridization, or hybridization histochemistry, allows us to visualize messenger ribonucleic acid (mRNA) and to draw conclusions about gene expression. These methods yielded very important new data and opened new avenues in the better understanding of structurefunction correlations. However, they also had some shortcomings. Light-microscopic immunocytochemistry and immunoelectron microscopy can only demonstrate intracellular hormone content; the results are qualitative and cannot be expressed quantitatively. In addition, the immunocytochemical study of hormone content of individual cells is often cumbersome and time-consuming, especially when the percentage of hormone-producing cells is low, when the morphological criteria for cell classification are inadequate, and when functional heterogeneity exists among individual cells. Electron microscopy can provide only indirect information on hormone synthesis and release. In situ hybridization combined with autoradiography requires a considerable amount of time and the results remain difficult to quantitate. Radioimmunoassay can express hormone secretion nLmerically but cannot relate it to the endocrine activity of individual cells. An elegant method, called the reverse hemolytic plaque assay (RHPA), seems to be suitable for overcoming some of these shortcomings.

The original hemolytic plaque assay was described by Jerne and Nordin in 1963 [5] for the detection of antibody-secreting cells. Since then it has undergone several modifications [3] and has been widely applied in immunological laboratories. The RHPA, which detects cells secreting antigens rather than antibodies, represents a major modification of the original technique. In this assay, protein A-coated erythrocytes are substituted for antibody-coated erythrocytes $[7,8]$. The mixture of protein A-conjugated erythrocytes and the cells whose secretion activity is investigated are incubated with a specific antiserum in a Cunningham slide chamber [1]. If the antigen, such as a hormone, neuropeptide, or neurotransmitter, is secreted from a cell, the released antigen combines with the specific antibody that is bound to the erythrocytes by protein $A$. If the indicator cells are coated with antigenantibody complexes, they undergo lysis with the addition of guinea pig complement. Thus, the cells secreting antigens can be demonstrated by a clear round spot or plaque, surrounded by an opaque layer of erythrocytes.

The significance of the RHPA is that it can be used to detect any kind of antigensecreting cells if an appropriate antibody is available. Consequently, the RHPA has been applied to a large number of cells that secrete immunoglobulins, albumin, alpha-fetoprotein, muramidase, T-cell factors, hormones, and the like [8]. The method for the demonstration of secretion of pituitary hormones by adenohypophyseal cells was introduced by Neill and Frawley [9]. They reported the detection of prolactin secretion by individual lactotrophs in a mixed culture of nontumorous rat adenohypophyseal cells [9]. Since this original publication, the method has been used chiefly in pituitary studies, including the investigation of rat and human pituitaries and pituitary adenomas.

Although the RHPA is a valuable method in endocrinology, there are some disadvantages that must be overcome, and caution is needed in the interpretation of the results [10]. The technique is time-consuming, and one must take into account that the antibody concentration applied in RHPA is much higher than that used in immunocytochemistry. Not only is this uneconomical, but it also 
may cause nonspecific hemolysis due to cross-reaction. Another problem may be that some complements used in RHPA may cause nonspecific hemolysis; therefore, these complements must be preabsorbed with sheep blood. To assure specificity of RHPA, several criteria must be fulfilled: Preabsorption of antiserum with antigen excess, omission of reagents such as antiserum or complement, or replacement of antiserum with normal rabbit serum must abolish plaque formation. It should also be noted that hemolytic plaques can be formed not only by secreted antigens but also by antigens released from damaged cells. Passive antigen leakage was confirmed by electron-microscopic studies [12].

The RHPA detects only those cells that actually secrete hormones. In this way it differs from immunocytochemistry, which can reveal those cells that contain the hormone but do not necessarily release it. The RHPA can be applied to study whether hormone secretion occurs in nonfunctioning endocrine tumors that have been considered to be unassociated with clinical or biochemical evidence of hormone excess [11]. The RHPA is quantitative, since the size of the plaque is related to the amount of hormone secreted by the cell. Thus, the percentage of plaque-forming cells and the size of plaques are quantitative indicators of hormone secretion.

Many tumors are heterogeneous. The RHPA can identify subpopulations of cells in the tumors that secrete a specific hormone [6]. Analysis of the percentage of plaqueforming cells and the size of plaques during exposure to a secretagogue at various concentrations allows the definition of its effect at the individual cell level; it can be established whether a secretagogue or inhibitor increases or decreases the number of secreting cells or the amount of hormone released by each cell [4]. One should keep in mind, however, that trypsin should not be used to disperse or harvest the cells in studying the effect of a secretagogue, for trypsin can damage the cell membrane.

The hemolytic reaction does not kill the cells. Thus, the RHPA can be applied sequentially (i,e, the same cells can be used to estimate variations over time in the amount of hormone secreted by individual cells, or one can investigate sequentially the effect of several stimulating or suppressing factors, or one may detect the cells secreting more than one hormone using a different antibody [2].

The sequential RHPA permits the study of plurihormonal tumors and helps to clarify whether more than one hormone is discharged from different cells or from the same cells. Furthermore, it also permits one to understand-at the single cell level-how secretagogues affect hormone release in monomorphous tumors.

When the evaluation of RHPA has been completed, several other techniques, such as immunocytochemistry, electron microscopy, and in situ hybridization, can be applied to the cells that have already been functionally classified by RHPA. These studies may provide a better understanding of the roles of gene expression, secretagogue binding and internalization, activation of a second messenger system, hormone synthesis, and the role of cytoskeleton in hormone secretion.

Immunocytochemistry may permit the detection of secretory cells or may allow the identification of plurihormonal cells by using different antibodies [6]. In situ hybridization may help us to understand better the relationship berween gene expression, hormone processing, and content and secretion at the single cell levels.

Electron-microscopic investigation can be undertaken on single plaque-forming cells [10]. This may give a deeper insight into structure-function correlations. The RHPA may clarify, especially if combined with electron-microscopy, the differences that may exist at organelle levels between nonsecretory cells (no plaque-forming cells) and secretory cells or between cells secreting a small amount of hormone (small plaque-forming cells) and those secreting a large amount of hormone (large plaque-forming cells) [12]. Immunoelectron microscopy can also be applied to single plaque-forming cells, providing additional information on structurefunction correlations in endocrine cells and tumors.

Autoradiography can also be combined with RHPA [10]. Receptor binding of the secretagogue can be measured using single cells with the help of the autoradiographic study of radiolabeled secretagogue. This method may be useful to confirm the secretion-receptor correlation. 
Application of the RHPA in the study of various endocrine tumors permits accurate quantitation of hormone secretion from a large number of cells and the classification of endocrine tumors into functional subpopulations. Furthermore, when combining the RHPA with other techniques, one can obtain a better understanding of function-structure correlations at both the microscopic and electron-microscopic levels in endocrine tumors. I believe that in future the RHPA will be applied to the study of several types of endocrine tumors. By this method, cells can be separated in various endocrine tumors, and their hormone release and the regulation of hormone secretion can be investigated.

Shozo Yamada, M.D., Ph.D. Department of Neurosurgery Toranomon Hospital 2-2-2 Toranomon Minato-ku Tokyo 105 Japan

\section{References}

1. Cunningham AJ, Szenberg A. Further improvements in the plaque technique for detecting single antibody-forming cells. Immunology 14:599-601, 1968 .

2. Frawley LS, Boockfod FR, Hoeffler JP. Identification by plaque assay of a pituitary cell type that secretes both growth hormone and prolactin. Endocrinology 116:734-737, 1985.

3. Gronowicz E, Coutinho A, Melchers F. A plaque assay for all cells secreting Ig of a given type or class. Eur J Immunol 6:588-590, 1976.

4. Hofland LI, Koetsveld PMV, Vroonhoven CCJV, Stefanko SZ, Lamberts SWJ. Heterogeneity of growth hormone $(\mathrm{GH})$ release by individual pituitary adenoma cells from acromegalic patients, as determined by the reverse hemolytic plaque assay: effects of SMS 201-995, GH releasing hormone and thyrotropin-releasing hormone. J Clin Endocrinol Metab 68:613-620, 1989.

5. Jerne $\mathrm{NK}$, Nordin AA. Plaque formation in agar by single antibody-producing cells. Science 24:405, 1963.

6. Lloyd RV, Anagnostou D, Cano M, Barkan AL Chandler W. Analysis of mammosomatotropic cells in normal and neoplastic human pituitary tissues by the reverse hemolytic plaque assay and immunocytochemistry. J Clin Endocrinol Metab 66:1103-1109, 1988.

7. Molinaro GA, Dray S. Antibody coated erythrocytes as a manifold probe for antigens. Nature 248:515-517, 1974.

8. Molinaro GA, Eby WC, Molinaro CA. The reverse plaque-forming cell assay. Methods Enzymol 73:326-338, 1981 .

9. Neil JD, Frawley LS. Detection of hormone release from individual cells in mixed populations using a reverse hemolytic plaque assay. Endocrinology 112:1135-1137, 1983.

10. Smith PF, Luque EH, Neill JD. Detection and measurement of secretion from individual neuroendocrine cells using a reverse hemolytic plaque assay. Methods Enzymol 124: 443-465, 1986.

11. Yamada S, Asa SL, Kovacs K, Muller P, Smith HS. Analysis of hormone secretion by clinically nonfunctioning human pituitary adenomas using the reverse hemolytic plaque assay. J Clin Endocrinol Metab 68:73-80, 1989.

12. Yamada $S$, Hattori A, Suzuki $T$, Aiba $T$, Asa SL, Kovacs K. Reverse hemolytic plaque assay: electron microscopic observation of plaqueforming single adenoma cells in $\mathrm{GH}$ producing adenomas. Submitted to Path Res Pract. 